\title{
A large database study of hospitalization charges and follow-up re-admissions in US lumbar fusion surgeries using a cellular bone allograft $(\mathrm{CBA})$ versus recombinant human bone morphogenetic protein-2 (rhBMP-2)
}

Bradley Wetzell ${ }^{1 *}$ (D, Julie B. McLean ${ }^{1}$, Mark A. Moore ${ }^{1}$, Venkateswarlu Kondragunta ${ }^{2}$ and Kimberly Dorsch ${ }^{3}$

\begin{abstract}
Background: The objective of this study was to retrospectively compare initial procedure and 12-month follow-up hospitalization charges and resource utilization (lengths of stay; LOS) for lumbar fusion surgeries using either recombinant human bone morphogenetic protein-2 (rhBMP-2) or a cellular bone allograft comprised of viable lineage-committed bone cells (V-CBA) via a large US healthcare system database. Potentially relevant re-admissions during the follow-up period were also assessed.

Methods: A total of 16,172 patients underwent lumbar fusion surgery using V-CBA or rhBMP-2, of whom 3503 (21.66\%) patients had follow-up re-admission data. Initial patient, procedure, and hospital characteristics were assessed to determine confounding factors. Multivariate regression modeling compared differences in hospitalization charges (in 2018 US dollars) and LOS (in days) between the groups, as well as incidences of potentially relevant re-admissions during the 12-month follow-up period.
\end{abstract}

Results: The adjusted mean initial procedure and 12-month follow-up hospital charges were significantly lower in the $V$-CBA group versus the rhBMP-2 group $(\$ 109,061$ and $\$ 108,315$ versus $\$ 160,191$ and $\$ 130,406$, respectively; $P<$ 0.0001 for both comparisons). This disparity remained in an ad hoc comparison of charges for initial single-level treatments only $(\mathrm{V}-\mathrm{CBA}=\$ 103,064$, rhBMP-2 $=\$ 149,620 ; P<0.0001)$. The adjusted mean initial LOS were significantly lower in the $V$-CBA group (3.77 days) versus the rhBMP-2 group (3.88 days; $P<0.0001$ ), but significantly higher for the cumulative follow-up hospitalizations in the 12-month follow-up period (7.87 versus 7.46 days, respectively; $P<0.0001)$. Differences in rates of follow-up re-admissions aligned with comorbidities at the initial procedure. Subsequent lumbar fusion rates were comparable, but significantly lower for V-CBA patients who had undergone single-level treatments only, in spite of V-CBA patients having significantly higher rates of initial (Continued on next page)

\footnotetext{
* Correspondence: brad_wetzell@lifenethealth.org

${ }^{1}$ Global Scientific Affairs and Clinical Engagement, LifeNet Healthø 1864 Concert Drive, Virginia Beach, VA 23453, USA

Full list of author information is available at the end of the article
}

(c) The Author(s). 2020 Open Access This article is licensed under a Creative Commons Attribution 4.0 International License, which permits use, sharing, adaptation, distribution and reproduction in any medium or format, as long as you give appropriate credit to the original author(s) and the source, provide a link to the Creative Commons licence, and indicate if changes were made. The images or other third party material in this article are included in the article's Creative Commons licence, unless indicated otherwise in a credit line to the material. If material is not included in the article's Creative Commons licence and your intended use is not permitted by statutory regulation or exceeds the permitted use, you will need to obtain permission directly from the copyright holder. To view a copy of this licence, visit http://creativecommons.org/licenses/by/4.0/ The Creative Commons Public Domain Dedication waiver (http://creativecommons.org/publicdomain/zero/1.0/) applies to the data made available in this article, unless otherwise stated in a credit line to the data. 
(Continued from previous page)

comorbidities that could negatively impact clinical outcomes.

Conclusions: The results of this study indicate that use of V-CBA for lumbar fusion surgeries performed in the US may result in substantially lower overall hospitalization charges versus rhBMP-2, with both exhibiting similar rates of 12-month re-admissions and subsequent lumbar fusion procedures.

Keywords: Cellular bone allograft, CBA, Economics, Lumbar fusion, Infuse, Recombinant human bone morphogenetic protein-2, rhBMP-2, ViviGen

\section{Introduction}

Lumbar spine disorders are among the most prevalent medical diagnoses across the globe [1] and spinal fusion surgeries are a common and historically successful intervention [2]. Autologous iliac crest bone grafts (ICBG) are traditionally preferred for these procedures due to their presumed potential to provide all three necessary properties for bone fusion (i.e., osteoconductivity, osteoinductivity, and osteogenicity) [3]. However, the supply of such material is limited, and the additional surgical procedure increases operative time, blood loss, risk of infection, and post-operative pain [4]. Additionally, the autograft quality may be limited by patient comorbidities [4]. Thus, alternatives have emerged to meet the need for grafting materials that circumvent the inherent drawbacks of ICBG.

Among these alternatives, recombinant human bone morphogenetic protein- 2 with a bovine collagen sponge scaffold (rhBMP-2; marketed as Infuse ${ }^{\mathrm{TM}}$ by Medtronic Inc., Memphis, TN), has been widely used since gaining approval by the US Food and Drug Administration in 2002 [5-7]. Although currently indicated in the spine solely for use in single-level anterior lumbar interbody fusion (ALIF) and single-level oblique lateral interbody fusion (OLIF) surgeries, up to $85 \%$ of its use is reported to be off-label $[5,8]$. Bone morphogenetic protein- 2 is part of a larger family of osteoinductive proteins known to induce mesenchymal stem cells to differentiate into bone-forming cells, such as osteoblasts [5]. However, rhBMP-2 alone is neither osteoconductive nor osteogenic and is thus often used in conjunction with other grafting materials. In spite of serious complications attributed to rhBMP-2 in the spine (e.g., wound complications, increased myelopathy/radiculopathy, and heterotopic ossification) $[9,10]$, several clinical studies have demonstrated that rhBMP-2 in lumbar fusion surgeries increases fusion rates compared to ICBG, while also decreasing fusion time and refusion rates [11]. However, rhBMP-2 remains relatively expensive $[12,13]$ and some third-party payers have become increasingly unwilling to reimburse for its prevalent off-label use, leading to downward trends in its overall use from all-time highs in 2009 [7, 14].

Cellular bone allografts (CBAs) are another alternative, which theoretically contain osteoinductive, osteoconductive, and osteogenic properties $[15,16]$. In particular, a more recent advanced CBA comprised of viable cryopreserved lineage-committed bone-forming cells (V-CBA; marketed as ViviGen $^{\oplus}$ by LifeNet Health ${ }^{\oplus}$, Virginia Beach, VA) uniquely emulates the benefits of ICBG without its inherent drawbacks [16-20]. Unlike rhBMP-2, V-CBA can be used for homologous repair of any bone defect throughout the body $[8,21]$. Clinical studies of spinal fusion surgeries using VCBA have thus far reported successful fusion outcomes [16, 17], and V-CBA is potentially less expensive than rhBMP-2. However, no research to date has compared these two grafts on any level.

Thus, the primary objective of this study was to compare initial procedure and 12-month follow-up hospitalization charges and resource utilization for lumbar fusion surgeries using rhBMP-2 versus V-CBA using data from a large nationwide US healthcare system. The secondary objective was to assess the incidence of potentially relevant readmissions during the follow-up period, including any subsequent lumbar fusion procedures.

\section{Materials and methods}

\section{Study design, data source, and participant selection}

This was a retrospective cohort study conducted using data from the Premier Healthcare Database (PHD; Premier Healthcare Solutions, Inc; Charlotte, NC). The PHD is a US hospital-based, service-level, all-payer database with a geographically diverse, nationwide footprint [22]. At the time of this study, the PHD contained standard discharge data for approximately 208 million unique patients from over 1000 hospitals. Data included patient demographics, disease status, and date-encoded billed services within daily service records. Within-system activities for a given patient were tracked across visits using a unique patient identification code, which did not contain personally identifiable information.

Data from the PHD are thus considered de-identified in accordance with the HIPAA Privacy Rule described in Title 45 of the US Code of Federal Regulations (CFR) Part 164.506(d)(2)(ii)(B) and are exempt from Institutional Review Board (IRB) oversight, as provided in 45 CFR 46.101(b)(4) [2, 22]. The protocol for this study was nonetheless submitted to Western IRB (Puyallup, WA), which confirmed its exempt status. 
The initial procedures for this study occurred from 1 October 2015 through 30 September 2018, with a 12month follow-up period for each patient extending through 30 September 2019. Data for patients meeting any of the following criteria were excluded from the PHD extract: patients not at least 18 years of age at the time of the initial procedure, patients from hospitals that did not continuously report to the PHD throughout the follow-up period, and patients who died during the initial admission.

From this extract, patients undergoing lumbar fusion procedures during the initial procedure period were isolated through a search of relevant ICD-10 procedure codes (i.e., ORGA\%, 0SG0\%, OSG1\%, and 0SG3\%). The resulting data subset was reviewed to determine search strings that would automatically isolate as many patients as possible who received either V-CBA or rhBMP-2 during the initial procedure, and the remaining data in the subset were manually reviewed for evidence of either graft. Data for patients who did not receive V-CBA or rhBMP-2, who received both V-CBA and rhBMP-2, or for whom the graft material could not be definitively determined were excluded from analysis.

Patients with all-cause follow-up re-admissions were identified by searching the final dataset for encounters designated as inpatient during the 12-month follow-up period. Importantly, these data did not include patients who may have received follow-up treatment outside of the Premier Healthcare System. Potentially relevant follow-up re-admissions were identified by searching the re-admission dataset for a predetermined list of diagnostic and procedural ICD-10 codes (see the following section).

\section{Study variables and statistical methods}

Patient, procedure, and hospital characteristics that were assessed at the initial procedure included age, sex, race, ethnicity, Charlson comorbidities, health insurance status, initial admission type, initial admission source, initial discharge status, cage insertion, multiple levels treated, hospital size, hospital teaching status, hospital population served, and hospital region. Charlson comorbidities were assessed using ICD-10 codes as described by Quan et al. [23], and Charlson Comorbidity Index (CCI) scores were calculated for each patient [24]. Cage insertion was categorized (with associated ICD-10 codes) as yes (0SG10A0, OSG10AJ, OSG13A0, OSG13AJ, OSG30A0, OSG30AJ, OSG33A0, OSG33AJ, OSG34A0, OSG23AJ, OSG03A0, OSG03AJ, OSG04A0, OSG04AJ, ORGA3A0, 0RGA3AJ, 0RGA4A0, and 0RGA4AJ) or no (all others) and multiple levels treated was categorized as yes (0SG1\%) or no (all others). Continuous variables were presented as means and standard deviations (SD) and categorical variables were presented as numbers and percentages of patients within each group (i.e., V-CBA and rhBMP-2). Preliminary analyses were conducted using two-sample $t$, Fisher's exact, Chi-square, and Wilcoxon rank-sum tests, as appropriate, to identify confounding factors in initial patient, procedure, and hospital characteristics between groups, which were then treated as covariates in the primary analyses.

For the primary objective, hospitalization charges and resource utilization were calculated using total charges for each patient (in 2018 US dollars) and reported lengths of stay (LOS; in days) for each patient, respectively, for hospitalizations during the initial procedure and cumulatively within the 12-month follow-up period for each patient. Crude means were reported for each group at each time period. Multivariate regression modeling was used to compare differences in hospitalization charges and LOS between the V-CBA and rhBMP-2 groups, adjusting for confounding factors identified in the preliminary analyses, and the resulting adjusted means and $95 \%$ confidence intervals (CIs) were presented.

For the secondary objective, potentially relevant followup re-admissions were assessed as the following procedural and diagnostic variables (and associated ICD-10 codes): subsequent lumbar fusion procedures (ORGA\%, 0SG0\%, 0SG1\%, and 0SG3\%), cardiac complications (I21.\%, I97.88, and I97.99), deep vein thrombosis (I80.00, I80.10, I80.209, I80.3, I80.219, I80.8, I80.9, I82.1, I82.220, I82.221, I82.419, I82.429, I82.439, I82.4Y9, I82.449, I82.499, I82.4Z9, I82.509, I82.599, I82.519, I82.529, I82.539, I82.5Y9, I82.549, I82.5Z9, I82.819, I82.890, and I82.91), hematoma (M96.84\%), nervous system complications (G03.8, G97.0, G97.81, and G97.82), pneumonia (J12.\%, J13.\%, J14.\%, J15.\%, J16.\%, J17.\%, J18.\%, and J95.851), pulmonary embolism (I26.\%, T80.0XXA, T81.718A, T81.72XA, T82.817A, T82.818A, I26.90, and I26.92), sepsis (T81.44\%), surgical-site infection (T81.41\%, $\mathrm{T} 81.42 \%, \mathrm{~T} 81.43 \%$, and $\mathrm{T} 81.49 \%$ ), and urinary tract infection (N39.0). The incidence of each complication during the 12-month follow-up period for each patient was presented as number and percentage of patients within each group, and comparisons between the V-CBA and rhBMP2 groups were conducted using Fisher's exact test.

Statistical analyses were performed using STATA software Version 15, (StataCorp, Inc., College Station, TX). Statistical assumptions were verified as appropriate for each statistical test, and significance was assessed at the 0.05 alpha level.

\section{Results}

\section{Participants}

The data-selection flow chart for this study is presented in Fig. 1. Application of the exclusion criteria to the study date ranges resulted in a PHD extract with data for 178,204 unique patients from 1085 hospitals and 


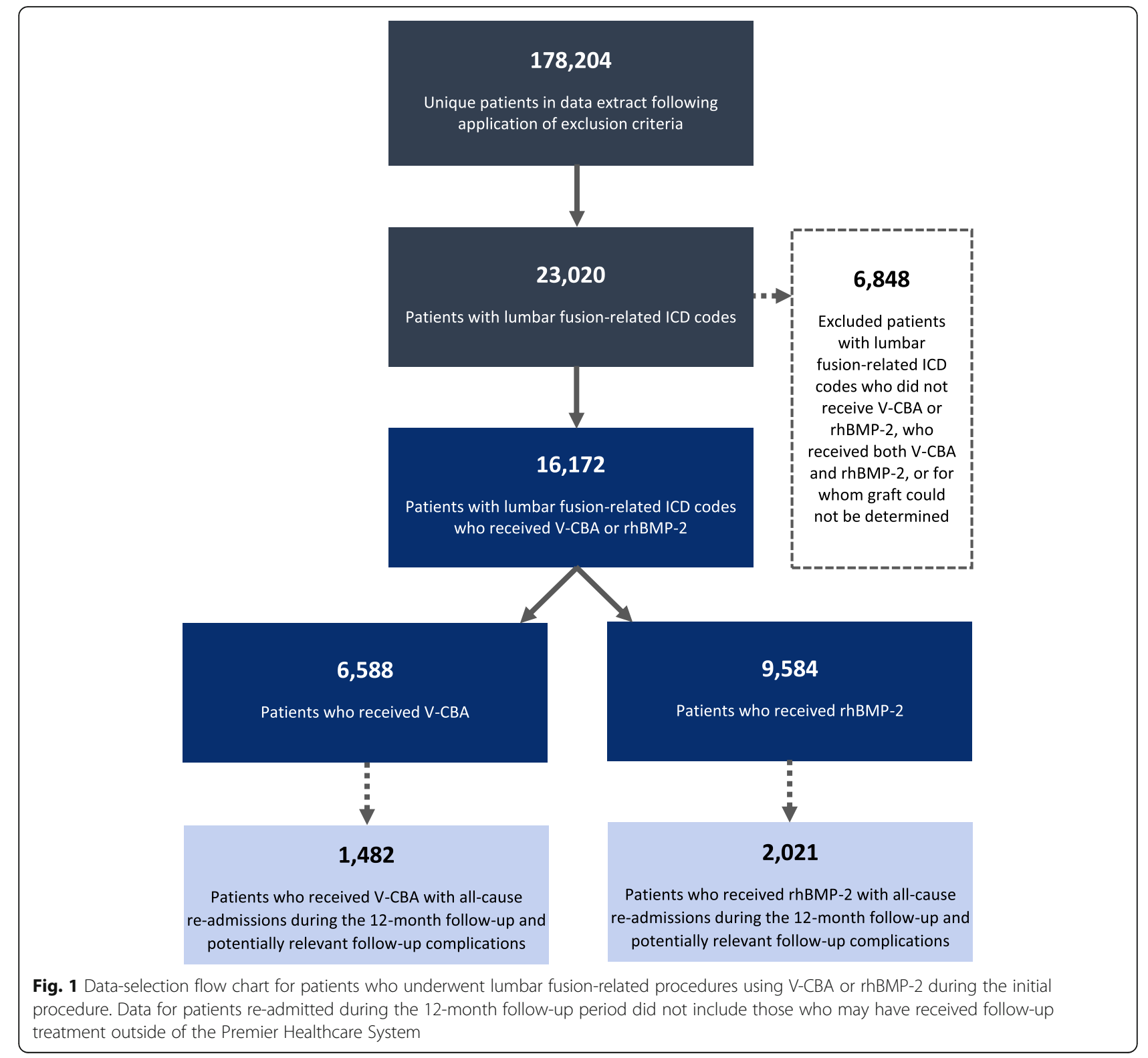

institutions. Within this extract, there were 23,020 patients with ICD-10 codes related to lumbar fusion surgeries during the initial procedure study period, of whom 6848 patients were excluded because they did not receive V-CBA or rhBMP-2, received both V-CBA and rhBMP-2, or the graft used could not be definitively determined. This process resulted in a final dataset for analysis with 16,172 patients from 172 hospitals and institutions, of whom 6588 patients received V-CBA and 9584 patients received rhBMP-2 during the initial procedure. All-cause inpatient re-admissions during the 12month follow-up period were identified for 1482 patients in the V-CBA group and 2021 patients in the rhBMP-2 group, which were then used to identify potentially relevant follow-up re-admissions.
Initial patient, procedure, and hospital characteristics The distributions and statistical comparisons of initial patient, procedure, and hospital characteristics are presented in Table 1 . The mean patient age $(\mathrm{SD})$ in each group was V-CBA $=60.86$ (13.13) and rhBMP-2 $=60.74$ (13.46) years and the majority of patients in each group were female $(\mathrm{V}-\mathrm{CBA}=55.45 \%$; rhBMP-2 $=55.32 \%)$, white $(\mathrm{V}-\mathrm{CBA}=84.79 \%$; rhBMP-2 $=87.80 \%)$, and nonHispanic $(\mathrm{V}-\mathrm{CBA}=84.82 \%$; rhBMP-2 $=90.90 \%)$. The mean CCI (SD) at the initial procedure was higher in the V-CBA group (0.92 [1.39]) than in the rhBMP-2 group (0.78 [1.20]). This difference resulted from significantly higher incidences in the V-CBA group for certain individual comorbidities, including (with $P$ values from Fisher's exact test) any malignancy (0.0001), cerebrovascular 


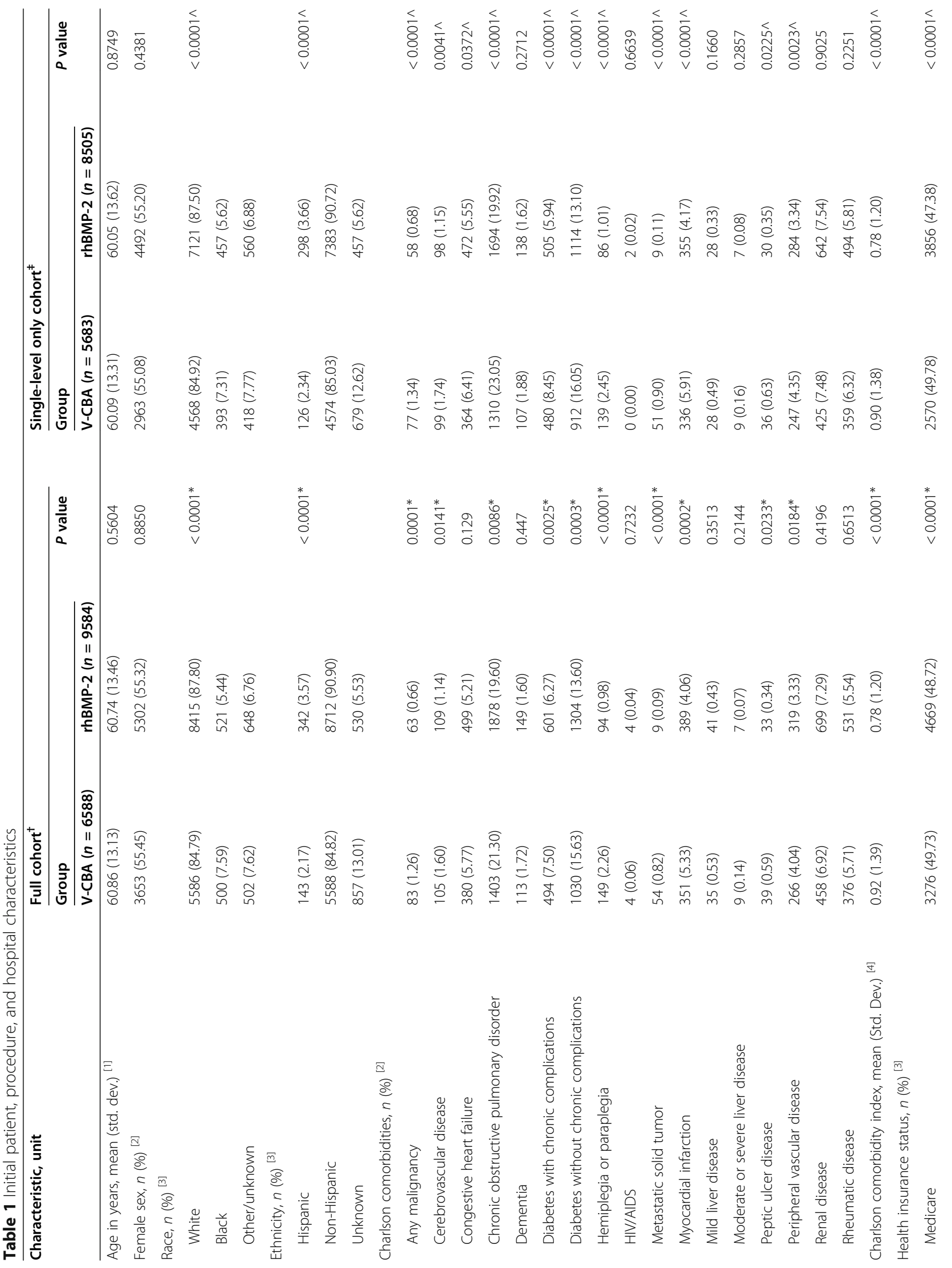




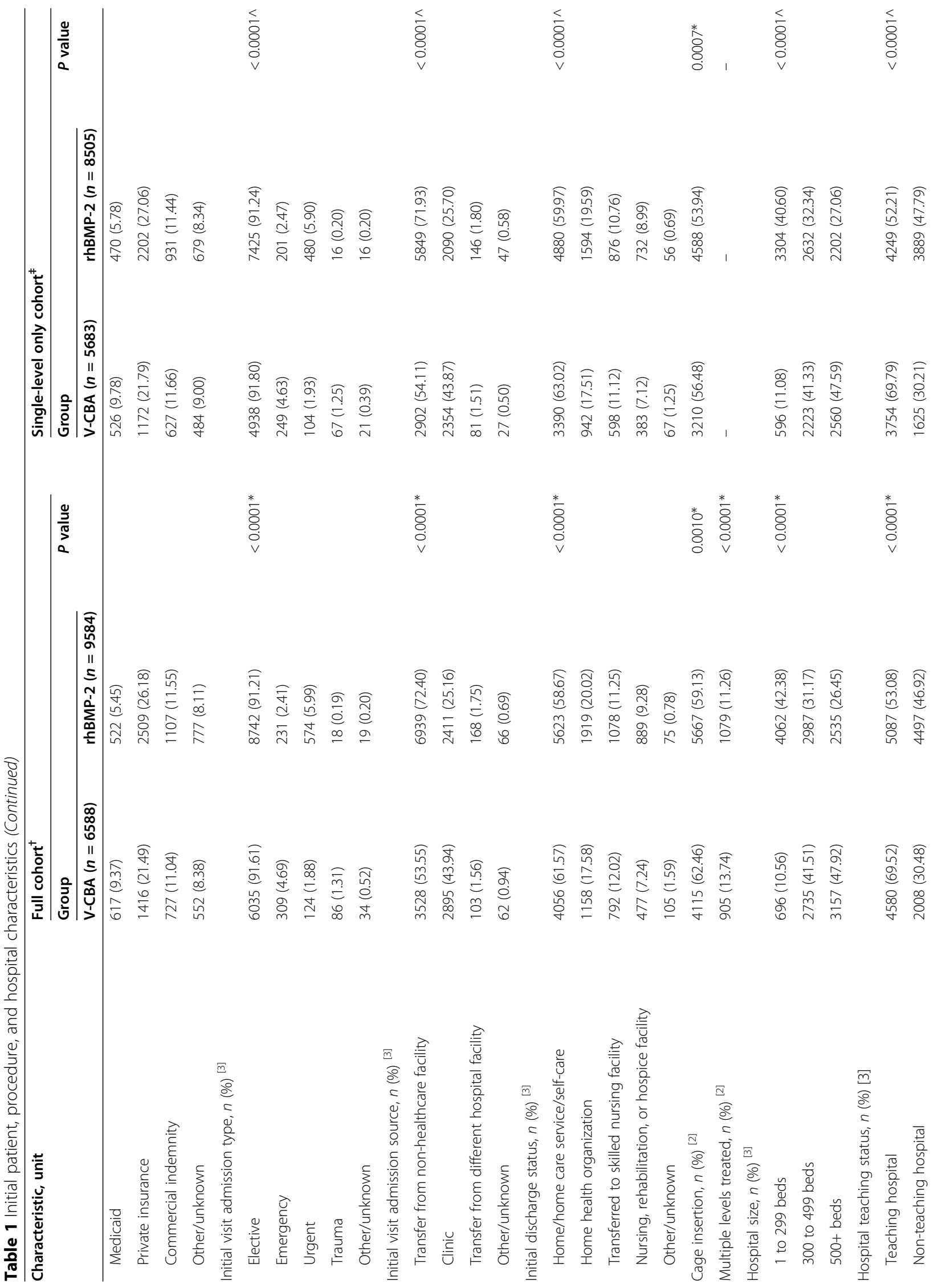




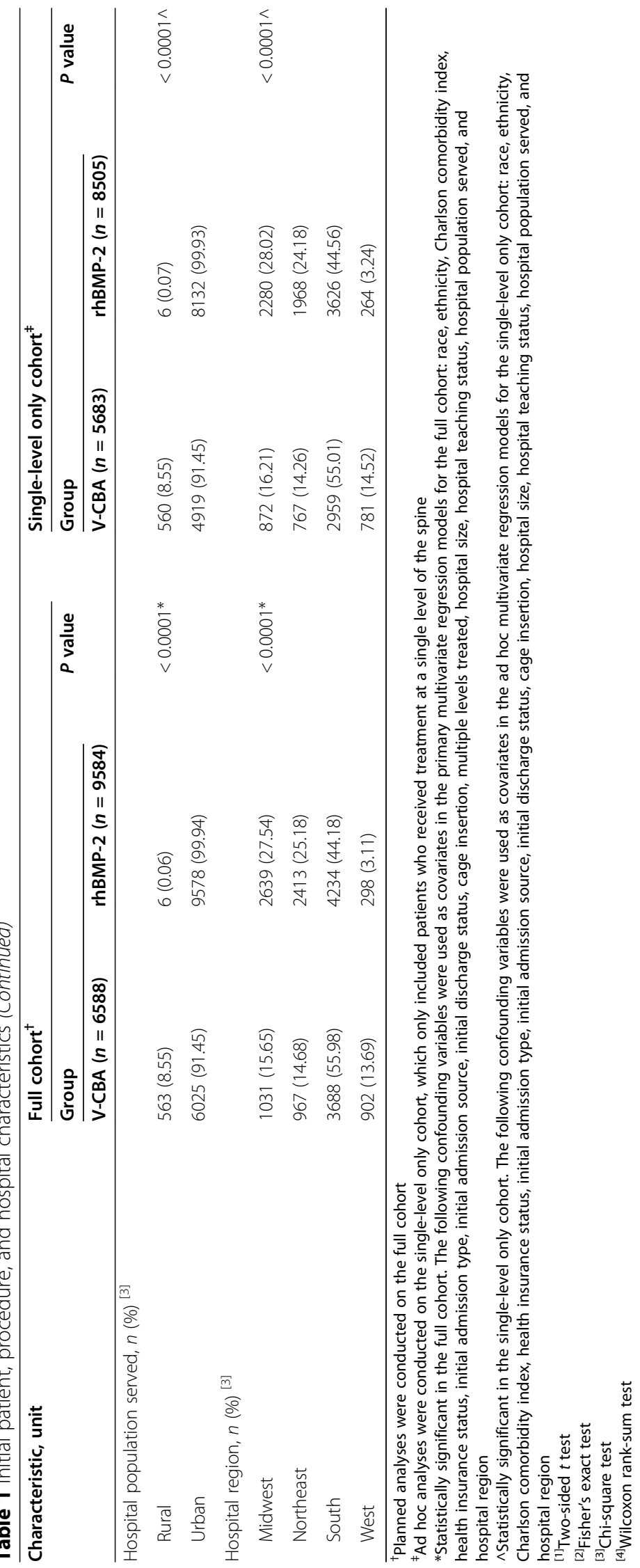


disease (0.0141), chronic obstructive pulmonary disorder (0.0086), diabetes with (0.0025) and without complications (0.0003), hemi- or paraplegia $(<0.0001)$, metastatic solid tumor $(<0.0001)$, myocardial infarction (0.0002), peptic ulcer disease (0.0233), and peripheral vascular disease (0.0184).

The most prevalent health insurance status in each group was Medicare $(\mathrm{V}-\mathrm{CBA}=49.73 \%$; $r$ BMMP-2 = $48.72 \%$ ), and the majority of initial visit admission types, initial admission sources, and initial discharge statuses were elective $(\mathrm{V}-\mathrm{CBA}=91.61 \%$; rhBMP-2 $=91.21 \%)$, transfer from non-healthcare facility $(\mathrm{V}-\mathrm{CBA}=53.55 \%$; rhBMP- $2=72.40 \%)$, and home/home care/self-care $(\mathrm{V}$ $\mathrm{CBA}=61.57 \%$; rhBMP-2 $=58.67 \%)$, respectively. In the $\mathrm{V}$-CBA group, $62.46 \%$ of the procedures included cage insertion (compared with 59.13\% in the rhBMP-2 group) and $13.74 \%$ of the procedures involved treatment of multiple levels (compared with $11.26 \%$ in the rhBMP-2 group).

For hospital size, the most prevalent in the V-CBA group was $500+$ beds (47.92\%), compared with 1 to 299 beds in the rhBMP-2 group (42.38\%). For both groups, the majority of hospitals were teaching hospitals (V-CBA $=69.52 \%$; rhBMP-2 $=53.08 \%$ ) serving urban populations $(\mathrm{V}-\mathrm{CBA}=91.45 \%$; rhBMP-2 $=99.94 \%)$, and the most prevalent US region represented in both groups was the South $(\mathrm{V}-\mathrm{CBA}=55.98 \%$; rhBMP-2 $=44.18 \%)$.

Statistical comparisons identified the following confounding initial patient, procedure, and hospital characteristics, which were treated as covariates in the multivariate regression models for the primary cost and resource utilization analyses: race, ethnicity, Charlson comorbidity index, health insurance status, initial admission type, initial admission source, initial discharge status, cage insertion, multiple levels treated, hospital size, hospital teaching status, hospital population served, and hospital region.

\section{Hospitalization charges and resource utilization}

The unadjusted mean hospitalization charges (SD) for the initial procedure were $\$ 118,917(\$ 77,459)$ for the VCBA group and $\$ 143,678(\$ 102,312)$ for the rhBMP-2 group $(P<0.0001$; Wilcoxon rank-sum test). Unadjusted hospitalization charges (SD) for the 12-month follow-up period were $\$ 108,763(\$ 120,353)$ for the V-CBA group and $\$ 120,449(\$ 131,041)$ for the rhBMP-2 group ( $P=$ 0.0477; Wilcoxon rank-sum test).

The adjusted mean initial procedure and follow-up hospital charges (95\% CIs) are presented in Fig. 2. After adjusting for confounding factors, the mean initial procedure and follow-up hospital charges (95\% CIs) remained significantly lower in the V-CBA group $(\$ 109$, $061[\$ 106,969-111,153]$ and $\$ 108,315$ [\$101,316-115, $314]$, respectively) versus the rhBMP-2 group $(\$ 160,191$ $[\$ 157,085-163,296]$ and $\$ 130,406$ [\$122,998-137,813], respectively). Given that this disparity may be skewed by the potential for higher cost variability between the groups during multiple-level treatments, an ad hoc analysis was performed on data only from patients who received single-level treatment $(\mathrm{V}-\mathrm{CBA}=86.26 \%$ and

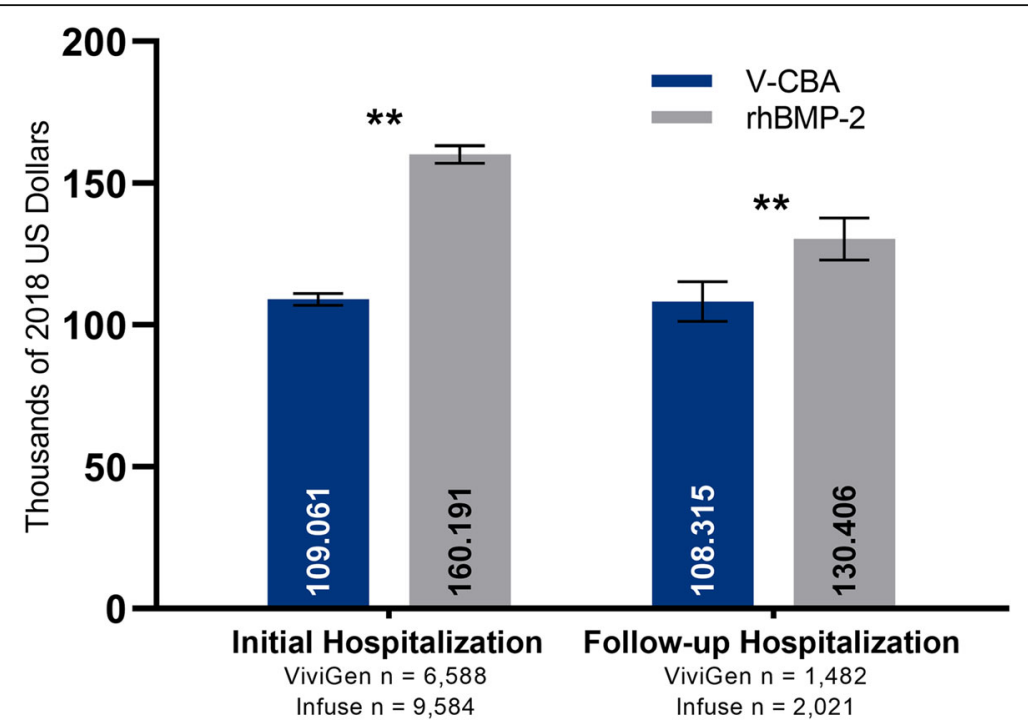

Fig. 2 Adjusted mean initial procedure and follow-up hospital charges $(95 \% \mathrm{Cls})$ were significantly lower with $\mathrm{V}$-CBA versus rhBMP-2. ${ }^{* *} P<$ 0.0001 . Multivariate regression models were adjusted with the following confounding factors as covariates: race, ethnicity, Charlson comorbidity index, health insurance status, initial admission type, initial admission source, initial discharge status, cage insertion, multiple levels treated, hospital size, hospital teaching status, hospital population served, and hospital region. Data for patients re-admitted during the 12-month followup period did not include those who may have received follow-up treatment outside of the Premier Healthcare System 
rhBMP-2 $=88.74 \%$ ). In theory, charges for the single-level procedures would be more standardized and could potentially provide for a better-aligned reference comparison. After adjusting for recalculated confounding factors for the ad hoc analysis (see single-level only cohort data, Table 1), mean initial procedure hospitalization charges (95\% CIs) for single-level treatments only remained significantly lower for the V-CBA group $(\$ 103,064[\$ 100$, 983-105,146]) versus the rhBMP-2 group $(\$ 149,620$ [\$146,469-152,772]; Fig. 3).

The mean initial procedure and follow-up LOS are presented in Table 2. The unadjusted LOS (SD) for initial procedure hospitalizations were 3.91 (4.48) days for the V-CBA group (range. 0-95 days) and 3.67 (3.30) days for the rhBMP-2 group (range. 0-81 days; not significant [ns]). Unadjusted cumulative LOS (SD) for the 12month follow-up period were 7.97 (12.38) days for the V-CBA group (range. 0-282 days) and 7.10 (8.52) days for the rhBMP-2 group (range. 0-92 days; ns).

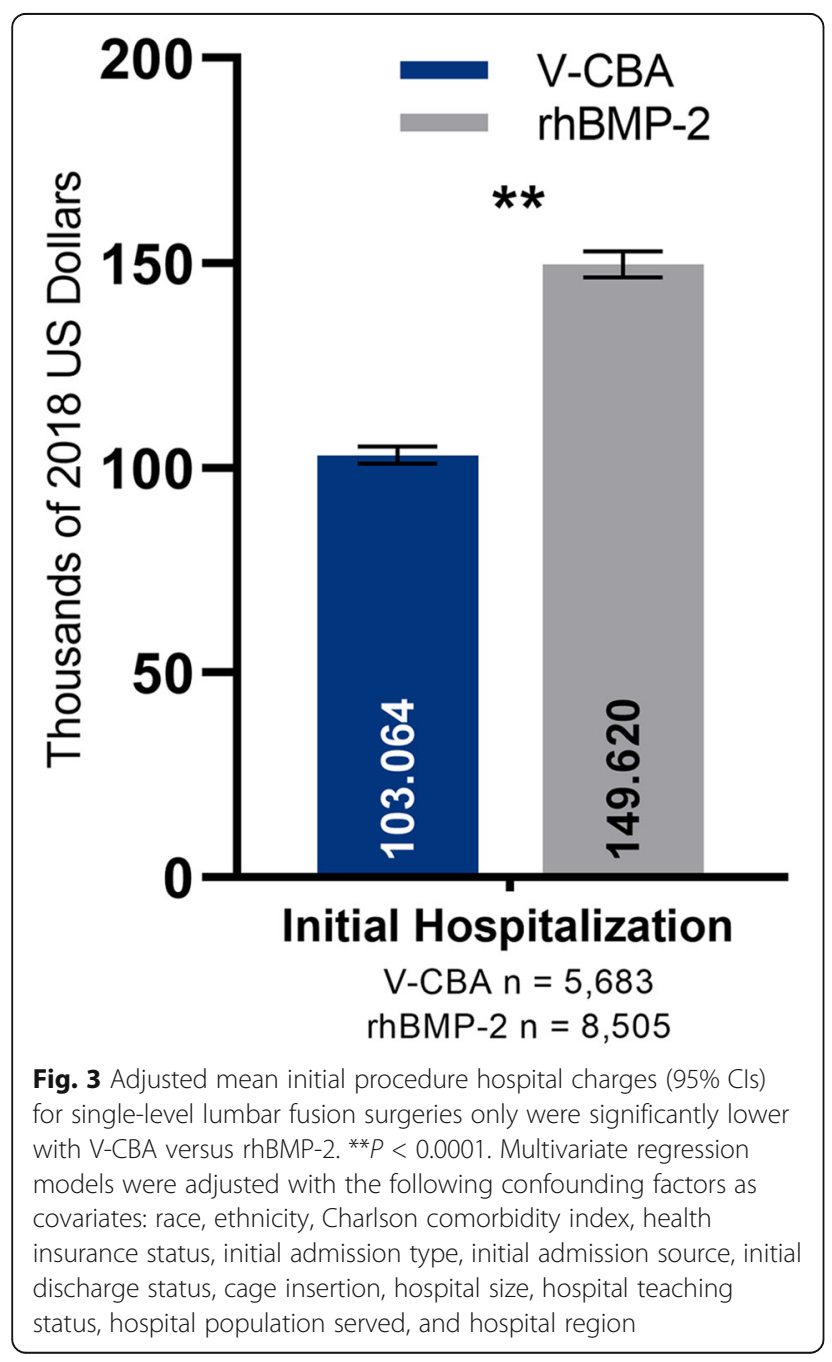

After adjusting for confounding factors, the mean initial procedure LOS $(95 \% \mathrm{CIs})$ were significantly lower in the V-CBA group (3.77 days [3.66-3.89 days]) versus the rhBMP-2 group (3.88 days [3.77-3.99 days]), but significantly higher for the cumulative follow-up hospitalizations in the 12-month period ( 7.87 days [7.21-8.53 days] versus 7.46 days [7.04-7.89 days], respectively). The notably wider variability in unadjusted follow-up LOS range for the V-CBA group (0-282 days) compared with the rhBMP-2 group (0-92 days) may have influenced the adjusted follow-up LOS results in spite of being treated as a covariate.

\section{Potentially relevant follow-up re-admissions}

The distributions and statistical comparisons of potentially relevant 12-month follow-up re-admissions are presented in Table 3. The 12-month incidence of cardiac complications was significantly higher in the V-CBA group $(0.71 \%)$ versus the rhBMP-2 group $(0.23 \%$; $P<$ $0.0001)$, as was the incidence of pneumonia $(1.21 \%$ versus $0.76 \%$, respectively; $P=0.0038$ ). However, these differences were consistent with those for individual comorbidities in the V-CBA group at the initial procedure (Table 1), which could not be controlled in this analysis. The incidences of all other re-admissions, including subsequent lumbar fusion procedures, deep vein thrombosis, hematoma, nervous system complications, pulmonary embolism, sepsis, surgical-site infection, and urinary tract infections were generally similar between the groups.

As with the cost analyses, the inclusion of multiple-level treatments could potentially skew the incidence of followup re-admissions between the groups. Therefore, a second ad hoc analysis was conducted on follow-up re-admissions among the single-level treatments only in an effort to standardize these comparisons. The incidence of cardiac complications remained significantly higher in the V-CBA group versus the rhBMP- 2 group $(0.44 \%$ vs $0.15 \%$, respectively; $P=0.0125$ ), which remained consistent with individual comorbidities in the V-CBA group for patients receiving single-level treatments only (Table 1 ). However, the incidence of subsequent lumbar fusion procedures was significantly lower among patients receiving V-CBA in this better-aligned comparison (3.66\% versus $4.56 \%$ in the rhBMP-2 group; $P<0.0001$ ). The incidences of all other re-admissions, including deep vein thrombosis, hematoma, nervous system complications, pneumonia, pulmonary embolism, sepsis, surgical-site infection, and urinary tract infections were generally similar between the single-level treatment groups.

\section{Discussion}

The primary objective of this study was to compare initial procedure and 12-month follow-up hospitalization 
Table 2 Mean hospital lengths of stay (full cohort)

\begin{tabular}{|c|c|c|c|c|c|c|}
\hline \multirow[t]{3}{*}{ No. days } & \multicolumn{3}{|l|}{ Initial procedure } & \multicolumn{3}{|l|}{ Follow-up^ } \\
\hline & \multicolumn{2}{|l|}{ Group } & \multirow[t]{2}{*}{$P$ value } & \multicolumn{2}{|l|}{ Group } & \multirow[t]{2}{*}{$P$ value } \\
\hline & V-CBA $(n=6588)$ & rhBMP-2 $(n=9584)$ & & V-CBA $(n=1482)$ & rhBMP-2 $(n=2021)$ & \\
\hline Unadjusted $^{\dagger}(\mathrm{SD})$ & $3.91(4.48)$ & $3.67(3.30)$ & 0.0948 & $7.97(12.38)$ & $7.10(8.52)$ & 0.0694 \\
\hline Adjusted $^{\ddagger}(95 \% \mathrm{Cl})$ & $3.77(3.66,3.89)$ & $3.88(3.77,3.99)$ & $<0.0001^{*}$ & $7.87(7.21,8.53)$ & $7.46(7.04,7.89)$ & $<0.0001^{*}$ \\
\hline
\end{tabular}

$\wedge$ Did not include patients who may have received follow-up treatment outside of the Premier Healthcare System

${ }^{\dagger}$ Wilcoxon rank-sum test

${ }^{\ddagger}$ Multivariate regression models were adjusted with the following confounds as covariates: race, ethnicity, Charlson comorbidity index, health insurance status, initial admission type, initial admission source, initial discharge status, cage insertion, multiple levels treated, hospital size, hospital teaching status, hospital population served, and hospital region

*Statistically significant

charges and resource utilization for lumbar fusion surgeries using rhBMP-2 versus V-CBA using data from a large nationwide US healthcare system. The secondary objective was to assess the incidence of potentially relevant re-admissions during the follow-up period, including any subsequent lumbar fusion procedures. The present data showed that hospitalization charges were significantly lower when V-CBA was used in US lumbar fusion surgeries versus rhBMP-2, with $\$ 51,130$ less in adjusted mean initial procedure charges, and $\$ 22,091$ less in adjusted mean follow-up hospitalization charges (Fig. 2).

Although the use of rhBMP-2 is almost universally reported to increase hospitalization costs $[6,12,13,25-27]$, the cause of these large disparities is unknown, as they cannot be explained by direct product costs alone. A definitive answer to this question is beyond the scope of this study; however, an exploration of potential causes is warranted, albeit speculative. The disparity is likely explained by a cluster of smaller contributing factors, including direct product cost, non-cost-effective use of rhBMP-2, and the expense of adjunct products and procedures sometimes used with rhBMP-2, such as demineralized bone matrix or platelet-rich plasma (PRP). This is in contrast to $\mathrm{V}$-CBA, which is similar to autograft in that the use of adjuncts is unnecessary.

Additional costs could also be attributed to operating room time required for preparation of rhBMP-2 ( $\geq 15 \mathrm{~min})$

Table 3 Incidence of potentially relevant 12-month follow-up re-admission

\begin{tabular}{|c|c|c|c|c|c|c|}
\hline \multirow[t]{3}{*}{ Re-admissions, $n(\%)^{[1]}$} & \multicolumn{3}{|c|}{ Full cohort $^{\dagger}$} & \multicolumn{3}{|c|}{ Single-level only cohort $^{*}$} \\
\hline & \multicolumn{2}{|l|}{ Group } & \multirow[t]{2}{*}{$P$ value } & \multicolumn{2}{|l|}{ Group } & \multirow[t]{2}{*}{$P$ value } \\
\hline & $\begin{array}{l}\text { V-CBA } \\
(n=6588)\end{array}$ & $\begin{array}{l}\text { rhBMP-2 } \\
(n=9584)\end{array}$ & & $\begin{array}{l}\text { V-CBA } \\
(n=5683)\end{array}$ & $\begin{array}{l}\text { rhBMP-2 } \\
(n=8505)\end{array}$ & \\
\hline $\begin{array}{l}\text { Patients with all-cause 12-month follow-up } \\
\text { re-admissions }{ }^{[2]}\end{array}$ & $1482(22.5)$ & $2021(21.08)$ & - & $971(17.1)$ & $1198(14.1)$ & - \\
\hline \multicolumn{7}{|c|}{ Re-admitted patients with potentially relevant procedures/diagnoses ${ }^{[3]}$} \\
\hline Subsequent lumbar fusion procedures & $623(9.46)$ & $831(8.67)$ & 0.0879 & $208(3.66)$ & $388(4.56)$ & $<0.0001^{*}$ \\
\hline Cardiac complications & $47(0.71)$ & $22(0.23)$ & $<0.0001 \wedge$ & $25(0.44)$ & $13(0.15)$ & $0.0125 \wedge$ \\
\hline Deep vein thrombosis & $6(0.09)$ & $3(0.03)$ & 0.1725 & $4(0.07)$ & $3(0.04)$ & 0.7073 \\
\hline Hematoma & $22(0.33)$ & $30(0.31)$ & 0.8878 & $13(0.23)$ & $20(0.23)$ & 0.5990 \\
\hline Nervous system complications & $17(0.26)$ & $14(0.15)$ & 0.1422 & $13(0.23)$ & $9(0.11)$ & 0.1990 \\
\hline Pneumonia & $80(1.21)$ & $73(0.76)$ & $0.0038 \wedge$ & $50(0.88)$ & $46(0.54)$ & 0.1432 \\
\hline Pulmonary embolism & $28(0.43)$ & $37(0.39)$ & 0.706 & $19(0.33)$ & $22(0.26)$ & 0.8748 \\
\hline Sepsis & $2(0.03)$ & $5(0.05)$ & 0.7081 & $0(0)$ & $1(0.01)$ & 1.0000 \\
\hline Surgical-site infection & $15(0.23)$ & $20(0.21)$ & 0.8634 & $8(0.14)$ & $9(0.11)$ & 1.0000 \\
\hline Urinary tract infections & $144(2.19)$ & $171(1.78)$ & 0.0727 & $81(1.42)$ & $93(1.09)$ & 0.6341 \\
\hline
\end{tabular}

${ }^{\dagger}$ Planned analyses were conducted on the full cohort

${ }^{\ddagger} \mathrm{Ad}$ hoc analyses were conducted on the single-level only cohort, which only included patients who received treatment at a single level of the spine *Statistically significant, Fisher's exact test

$\wedge$ Statistically significant, Fisher's exact test. Notably, differences observed in follow-up diagnoses between groups corresponded with significant comparisons in related initial Charlson comorbidities within each cohort (Table 1)

${ }^{[1]}$ All percentages were based on the total number of patients within each cohort who received V-CBA or rhBMP-2 during the initial procedure

${ }^{[2]}$ Patients with more than one re-admission were counted only once. Did not include patients who may have received follow-up treatment outside of the Premier Healthcare System

${ }^{[3]}$ Except subsequent lumbar fusion procedures, repeats of the same diagnosis were counted only once. Did not include patients who may have received followup treatment outside of the Premier Healthcare System 
versus $\mathrm{V}-\mathrm{CBA}(<5 \mathrm{~min})$, as well as that required for preparation of rhBMP-2 adjuncts (e.g., spinning/prep of PRP). A study by Hall and colleagues of multi-level instrumented posterolateral fusion (IPLF) surgeries using V-CBA reported a mean operative time of $211.1( \pm 87.3)$ min with an average of 4.1 levels treated [16]. Yet Glassman and colleagues reported a mean operative time of $248( \pm 58.5) \mathrm{min}$ with IPLF surgeries using rhBMP-2 (average 1.96 levels treated) and $270( \pm 33.6) \mathrm{min}$ in those using ICBG (average 1.98 levels treated) [26]. As noted by Hall, the use of VCBA thus corresponded with an average of 37- and 59-min faster surgeries than with rhBMP-2 and ICBG, respectively [16], despite the difference in number of levels treated. Such disparities in operative time could potentially contribute to exponential differences in cost.

Another potential contributor may be procedure complexity, as surgeons may default to rhBMP-2 in more complex cases. To this end, the present data could only differentiate between single- and multiple-level surgeries, and multiple-level surgeries have substantially wider variation in costs, particularly with three or more levels of treatment. We hypothesized that such surgeries may have had undue influence on these data, in spite of being treated as covariates in the main analysis. As such, an ad hoc cost analysis was conducted using only charge data from single-level fusion procedures, which involved $86.26 \%(n=5683)$ and $88.74 \%(n=8505)$ of V-CBA and rhBMP-2 patients, respectively (Fig. 3). In theory, singlelevel cases should be more standardized. Yet, even under these more-aligned conditions, the adjusted mean initial procedure hospitalization charges remained significantly lower in the V-CBA group with $\$ 46,556$ less charges. These results suggest that procedure complexity does not contribute substantially to the cost disparity.

The difference in initial procedure charges may also be partially influenced by the statistically significant increase of 0.11 days in adjusted mean initial procedure LOS found in the rhBMP-2 group (Table 2). However, the potential influence of this factor on charges was not reflected in the cumulative follow-up LOS, for which a significant mean increase of 0.41 days was observed in the V-CBA group, in spite of the $\$ 22,091$ cost differential during this period. Notably, the unadjusted LOS range in the V-CBA group was much wider (0-282 days) than in the rhBMP-2 group (0-92 days), which likely contributed to these results and may be related to the significantly higher initial CCI for this group. However, given such small disparities at both time points, it is difficult to attribute any further clinical or practical significance to these results.

Regarding clinical outcomes, our analysis was restricted to hospitalizations within the Premier Healthcare System and, therefore, 12-month follow-up readmissions may be underestimated. However, it is reasonable to expect that the proportion of patients seeking treatment outside of the Premier Healthcare System was evenly distributed between the groups. It is also important to note that, unlike with the primary cost and LOS analyses, the binary nature of these data prevented control of the confounding baseline variables. Thus, their interpretation requires consideration of the confounding variables and, in particular, the individual Charlson comorbidities.

Accordingly, although the majority of potentially relevant 12-month re-admissions were similar between the groups, significantly higher rates of cardiac complications and pneumonia were observed in the V-CBA group versus the rhBMP-2 group (Table 3 ). However, although it was not possible to definitively determine relationships between specific comorbidities and follow-up diagnoses within each patient, these differences in follow-up diagnoses corresponded to the significantly higher prevalence of related pre-existing diagnoses in the V-CBA group (Table 1), including cerebrovascular disease, chronic obstructive pulmonary disorder, diabetes with chronic complications, myocardial infarction, and peripheral vascular disease. This trend remained in the ad hoc analysis of single-level treatment only, with only the cardiac complications remaining significant (Table 3). Therefore, the differences in 12-month followup re-admissions between V-CBA and rhBMP-2 are expected and align with corresponding differences in initial comorbidities.

The presence of malignancy and metastatic solid tumor in both groups during the initial procedure is worth noting, as these comorbidities are contraindicated with rhBMP-2 [28]. V-CBA can be used in patients with cancer, although it is not recommended when the patient is considered immunocompromised, such as may occur when actively receiving treatment (e.g., chemotherapy or radiation). However, it was not possible to determine if such treatments were received concomitantly in the present data. Further, a follow-up analysis revealed that the presence of these comorbidities did not appear to substantially alter rates of re-admission for either of the V-CBA or rhBMP-2 groups, as only one such patient was re-admitted (from the rhBMP-2 group for urinary tract infection; data not shown).

There were no significant differences in rates of subsequent lumbar fusion procedures between the V-CBA and rhBMP-2 groups for the full cohort. However, these rates were significantly lower in the V-CBA group among patients receiving single-level treatments only (Table 3). The low rate of subsequent lumbar fusion with rhBMP-2 is a frequently cited benefit over ICBG and a principal justification of its cost [11, 13, 27]. As such, the performance of V-CBA in this study is notable, especially in light of the substantially lower average 
initial hospitalization charges observed for V-CBA patients, and in spite of the higher level of initial comorbidities in this group.

Large database studies have inherent limitations. For instance, the data from the PHD reflect the dollar amount that was charged for patient services, which may not reflect the final cost to the patient or third-party claims paid to the hospital or provider. Those final charges would only be available to the hospitals and are beyond the scope of this study. Further, although this study provides access to high-quality economic and clinical data, some potentially relevant patient and procedure details were unavailable, such as extended medical histories, surgical approaches used, or fusion outcomes. Increased granularity may have helped differentiated factors affecting clinical outcomes or charges. As well, some patients may have received followup treatment outside of the Premier Healthcare System, making their data unavailable. Additionally, the grafting material used during the initial procedure was collected as a subjective text string and, in some cases, was not sufficient to definitively determine the material used. Thus, some patients with data relevant to V-CBA or rhBMP-2 may have been inadvertently excluded. Finally, the present data represented economic and clinical information from US hospitals only and thus did not permit characterization for other regions.

\section{Conclusions}

The results of this study indicate that use of V-CBA for lumbar fusion surgeries performed in the US may result in substantially lower overall hospitalization charges versus rhBMP-2, with both exhibiting similar rates of 12month re-admissions and subsequent lumbar fusion procedures.

\section{Abbreviations \\ ALIF: Anterior lumbar interbody fusion; CBA: Cellular bone allograft; CCI: Charlson comorbidity index; CFR: US code of federal regulations; Cl: Confidence interval; ICBG: Iliac crest bone grafts; IPLF: Instrumented posterolateral fusion; IRB: Institutional review board; LOS: Lengths of stay; OLIF: Oblique lateral interbody fusion; PHD: Premier healthcare database; PRP: Platelet-rich plasma; rhBMP-2: Infuse recombinant human bone morphogenetic protein-2 with a bovine collagen sponge scaffold; SD: Standard deviation; V-CBA: ViviGen cellular bone allograft}

\section{Authors' contributions}

All authors participated in the data analysis, were major contributors to the writing of the manuscript, and read and approved the final manuscript for publication.

\section{Authors' information}

BW, JBM, MAM, Global Scientific Affairs and Clinical Engagement, LifeNet Health ${ }^{\oplus}$, Virginia Beach, VA. VK, Independent biostatistical consultant, Biomarker Statistics, LLC. KD, Global Clinical Affairs, LifeNet Health», Virginia Beach, VA.

\section{Availability of data and materials}

The data that support the findings of this study are available from Premier Healthcare Solutions, Inc. (Charlotte, NC), but restrictions apply to the availability of these data, which were used under license for the current study, and so are not publicly available. Data are however available from the authors upon reasonable request and with permission of Premier Healthcare Solutions, Inc.

\section{Ethics approval and consent to participate}

Patient data for this study were considered de-identified in accordance with the HIPAA Privacy Rule described in Title 45 of the US Code of Federal Regulations (CFR) Part 164.506(d)(2)(ii)(B) and are exempt from Institutional Review Board (IRB) oversight, as provided in 45 CFR $46.101(\mathrm{~b})(4)[2,22]$. The protocol for this study was nonetheless submitted to Western IRB (Puyallup, WA), which confirmed its exempt status.

\section{Competing interests}

BW, JBM, MAM, and KD are employees of LifeNet Health ${ }^{\oplus}$, a non-profit organization, which funded the study. VK was an independent biostatistical consultant for LifeNet Health ${ }^{\oplus}$ in the preparation of this manuscript. However, potential bias was minimized through a study design where real-world data were obtained from a third-party database offered by Premier Healthcare Solutions, Inc. (Charlotte, NC), which allows for evidence- and population-based analyses of drugs, devices, other treatments, disease states, epidemiology, resource utilization, healthcare economics, and clinical outcomes.

\section{Author details}

${ }^{1}$ Global Scientific Affairs and Clinical Engagement, LifeNet Health ${ }^{\oplus}, 1864$ Concert Drive, Virginia Beach, VA 23453, USA. Biomarker Statistics, LLC, San Diego, CA, USA. ${ }^{3}$ Global Clinical Affairs, LifeNet Health ${ }^{\oplus}$, Virginia Beach, VA, USA.

Received: 25 August 2020 Accepted: 9 November 2020

Published online: 19 November 2020

\section{References}

1. Buser Z, Ortega B, D'Oro A, et al. Spine degenerative conditions and their treatments: national trends in the United States of America. Glob Spine J. 2018;8(1):57-67.

2. Rosenthal NA, Heinrich $\mathrm{KH}$, Chung J, Yu H. Cost and hospital resource utilization of Staphylococcus aureus infection post elective posterior instrumented spinal fusion surgeries in US Hospitals: a retrospective cohort study. Spine (Phila Pa 1976). 2019;44(9):637.

3. Khan SN, Cammisa FP Jr, Sandhu HS, Diwan AD, Girardi FP, Lane JM. The biology of bone grafting. J Am Acad Orthop Surg. 2005;13(1):77-86.

4. Younger EM, Chapman MW. Morbidity at bone graft donor sites. J Orthop Trauma. 1989:3(3):192-5.

5. Lykissas M, Gkiatas I. Use of recombinant human bone morphogenetic protein-2 in spine surgery. World J Orthop. 2017;8(7):531.

6. Cahill KS, Chi JH, Day A, Claus EB. Prevalence, complications, and hospital charges associated with use of bone-morphogenetic proteins in spinal fusion procedures. Jama. 2009;302(1):58-66.

7. Lao L, Cohen JR, Lord EL, Buser Z, Wang JC. Trends analysis of rhBMP utilization in single-level posterior lumbar fusion (PLF) in the United States. Eur Spine J. 2016;25(3):783-8.

8. Walker B, Koerner J, Sankarayanaryanan S, Radcliff K. A consensus statement regarding the utilization of BMP in spine surgery. Curr Rev Musculoskelet Med. 2014;7(3):208-19.

9. Epstein NE. Complications due to the use of BMP/INFUSE in spine surgery: the evidence continues to mount. Surg Neurol Int. 2013;4(Suppl 5):S343.

10. Fu R, Selph S, McDonagh M, et al. Effectiveness and harms of recombinant human bone morphogenetic protein-2 in spine fusion: a systematic review and meta-analysis. Ann Intern Med. 2013;158(12):890-902.

11. Zhang $H$, Wang $F$, Ding $L$, et al. A meta analysis of lumbar spinal fusion surgery using bone morphogenetic proteins and autologous iliac crest bone graft. PLoS One. 2014;9(6):e97049.

12. Alvin MD, Derakhshan A, Lubelski $D$, et al. Cost-utility analysis of 1-and 2level dorsal lumbar fusions with and without recombinant human bone morphogenic protein-2 at 1-year follow-up. J Spinal Disord Techn. 2016; 29(1):E28-33.

13. Jain A, Yeramaneni $\mathrm{S}$, Kebaish KM, et al. Cost-utility analysis of rhBMP-2 use in adult spinal deformity surgery. Spine (Phila Pa 1976). 2020;45(14):1009-15.

14. Epstein NE. Pros, cons, and costs of INFUSE in spinal surgery. Surg Neurol Int. 2011;2:10. 
15. Magnus MK, Iceman KL, Roukis TS. Living cryopreserved bone allograft as an adjunct for hindfoot arthrodesis. Clin Podiatr Med Surg. 2018;35(3):295-310.

16. Hall JF, McLean JB, Jones SM, Moore MA, Nicholson MD, Dorsch KA. Multilevel instrumented posterolateral lumbar spine fusion with an allogeneic cellular bone graft. J Orthop Surg Res. 2019;14(1):372.

17. Corso KA, Etter K, Menzie AM, Bhattacharyya S, Pracyk JB. Characteristics and rate of subsequent lumbar spine fusion surgery among patients treated with allogenic cellular bone matrix for posterior lumbar spinal fusion. Value in Health. 2018;21:S106.

18. Birmingham E, Niebur G, McHugh PE. Osteogenic differentiation of mesenchymal stem cells is regulated by osteocyte and osteoblast cells in a simplified bone niche. Eur Cells Mater. 2012;23:13-27.

19. Ghanaati S, Unger RE, Webber MJ, et al. Scaffold vascularization in vivo driven by primary human osteoblasts in concert with host inflammatory cells. Biomaterials. 2011;32(32):8150-60.

20. Tortelli F, Tasso R, Loiacono F, Cancedda R. The development of tissueengineered bone of different origin through endochondral and intramembranous ossification following the implantation of mesenchymal stem cells and osteoblasts in a murine model. Biomaterials. 2010;31(2):242-9.

21. Center for Biologics Evaluation and Research, Center for Devices and Radiological Health, Office of Combination Products. Regulatory considerations for human, cells, tissues, and cellular and tissue-based products: minimal manipulation and homologous use. Guidance for Industry and Food and Drug Administration Staff. In: US Department of Health and Human Services, Food and Drug Administration, eds. December ed 2017.

22. Premier Healthcare Database: Data that informs and performs. 2018. Available at: https://products.premierinc.com/downloads/ PremierHealthcareDatabaseWhitepaper.pdf. Accessed September 2018.

23. Quan $\mathrm{H}$, Sundararajan V, Halfon P, et al. Coding algorithms for defining comorbidities in ICD-9-CM and ICD-10 administrative data. Med Care. 2005; 43(11):1130-9.

24. Charlson M, Szatrowski TP, Peterson J, Gold J. Validation of a combined comorbidity index. J Clin Epidemiol. 1994;47(11):1245-51.

25. Glassman SD, Carreon LY, Campbell MJ, et al. The perioperative cost of Infuse bone graft in posterolateral lumbar spine fusion. Spine J. 2008;8(3): 443-8.

26. Glassman SD, Carreon LY, Djurasovic M, et al. RhBMP-2 versus iliac crest bone graft for lumbar spine fusion: a randomized, controlled trial in patients over sixty years of age. Spine (Phila Pa 1976). 2008;33(26):2843-9.

27. Safaee MM, Dalle Ore CL, Zygourakis CC, Deviren V, Ames CP. Estimating a price point for cost-benefit of bone morphogenetic protein in pseudarthrosis prevention for adult spinal deformity surgery. J Neurosurg Spine. 2019;30(6):814-21.

28. Medtronic Sofamor Danek USA Inc. Infuse bone graft: instructions for use. 2020. Document \#0381204E Rev.D. Available from: http://manuals. medtronic.com/content/dam/emanuals/spinal/0381204E_Infuse_eManual_ revD.pdf. Accessed August 2020.

\section{Publisher's Note}

Springer Nature remains neutral with regard to jurisdictional claims in published maps and institutional affiliations.

Ready to submit your research? Choose BMC and benefit from:

- fast, convenient online submission

- thorough peer review by experienced researchers in your field

- rapid publication on acceptance

- support for research data, including large and complex data types

- gold Open Access which fosters wider collaboration and increased citations

- maximum visibility for your research: over $100 \mathrm{M}$ website views per year

At $\mathrm{BMC}$, research is always in progress.

Learn more biomedcentral.com/submissions 